\title{
SPHMS : Smart Patient m-Healthcare Monitoring System with NFC and IOT
}

\author{
Hoda Ramin Hossein \\ Computer Department, \\ University of Pune, AISSMS, \\ $\mathrm{COE}$ \\ Pune, Maharashtra 411001, \\ India
}

\begin{abstract}
In the latest technology, NFC(Near Field Communication) is a recently growing technology for communication for short range which is aimed to augment existing near field technologies such as RFID(Radio Frequency Identification). In this paper, we have to propose a system which describes how an android application is used as our own NFC tag writer to write patient unique id in NFC tag. Doctors using NFC enabled smart phones to retrieve patient information when placed near NFC tag. This proposed system uses an android smart phone which automatically monitors the patients status and keeps the doctor/nurse updated and maintained through logs that are stored on a cloud server and also alerts are generated through /Email in case of emergency/detection of death of any patient. This system helps in reducing the paper work required during the registration of the patient and also eliminates the disadvantages of using RFID based system.
\end{abstract}

Keywords: NFC tags, web server, NFC enabled smart phone, Android application.

\section{INTRODUCTION}

An important requirement now-a-days for medical service informalization is the establishment and improvement of doctor-patient interaction system. Especially in today's era where the mobile communication technology develops rapidly, it is a challenge to make the mobile web of full use to make up for the time and distance gap between doctors and patients to provide fast and adequate medical services or not becomes an important factor to measure hospital's competitive ability. Hospitals and clinics are increasingly turning to modern technology to facilitate treatment and optimize patient management

In current technology enabled world, there is rapid change and the status-quo is constantly disrupted. Internet Of Things (IOT) is one such field where disruption is taking place right now and has the potential to change the way healthcare is delivered. IOT does not have any standard definition, as per Gartner, "IOT is a network of physical objects that contain embedded technology to communicate and sense or interact with their internal states or the external environment". People and things can be easily connected to each other at anytime, anyplace with the help of using any path or network and any service with the help of IOT.

There is one other technology emerging now-a-days which is Near Field Communication (NFC). NFC is known to be a wireless technology used for communication in a short range of four to ten centimetres. NFC is based on RFID technology which by using a reading device can be used to read information from a tag. By working in three different modes NFC provides excellent security and privacy. These modes are Read/write mode, Peer to peer mode, Card emulation mode.
1. Card Emulation mode:

An NFC device in a card emulation mode can replace a contactless card or a tag. This will enable NFC devices to be used with existing contactless card infrastructure in applications such as access control, payments and ticketing.

\section{Reader/Writer mode:}

An NFC enabled device is able to read and write to NFC and many contactless cards. For example, if a NFC tag is attached to a poster, the NFC Smartphone can "tap" the tag to access the information stored in the tag easy and conveniently.

3.Peer to Peer mode: A Peer to peer mode enables two NFC devices to share data between them. Here, the NFC is used to negotiate the optimal communication protocol and transfer authentication data for secondary protocol. Then the file or data is sent over the high capacity protocol(i.e Bluetooth, EiFi etc).

In this paper, we have proposed a system which describes how an android application is used as our own NFC tag writer to write patient unique id in NFC tag. This will help in reducing the paper work that is required during the registration when a patient is admitted in the hospital. Doctors will be using NFC enabled smart phones to retrieve patient information when placed near NFC tag. This system uses a smart phone which automatically monitors the patients status and keeps the doctor/nurse updated and maintained through logs that are stored on a cloud server and also alerts are generated through SMS/Email in case of emergency/detection of death of any patient. This system helps in reducing the paper work required during the registration of the patient and also eliminates the disadvantages of using RFID based system. 


\section{RELATED WORK}

\subsection{Literature Survey}

The existing system in healthcare involves manual intervention where there is note taking, updating the notes to the computer and maintaining the records under a unique id assigned to every patient. This process is usually very slow and error prone due to which there is latency between data gathering and information accessibility.

"PSMPA: Patient Self-controllable \& Multi-level Privacy Preserving cooperative authentication in distributed $\mathrm{m}$ healthcare cloud computing system"[1] has introduced security in $\mathrm{m}$-Healthcare by implementing a three tier architecture and providing security on the cloud where all the data of the patient is stored.

In recent work, "Design and Implementation of Doctor-Patient system based on android"[2] introduced m-health and Android platform which was designed to achieve a set of doctor-patient interaction system based on android. One other system A wireless ECG monitoring system[2] was implemented which integrated novel capacitive ECG sensors.

NFC is playing an important role, in "NFC-based hospital Real-Time Patient management system"[8], they have used NFC technology where NFC smart tag or wrist band is given to each patient during the time of registration. Then NFC encoded devices can be used to read the information from the patient without causing any inconvenience at any time.

In other work[9], they have used an android application to view the patients data with the help of an open source platform. This system has helped the doctor's in monitoring and viewing the patient's data through a mobile device.

\subsection{Existing System}

In our real time system, manual intervention is required i.e when a patient is admitted the registration of the patient is done manually which is more tedious and error prone job. Also when a patient is in critical condition, no alerts are generated which may sometimes cause loss of life. Also doctors don't have a provision of remote monitoring.

Before NFC, the similar device used was RFID. With using RFID the cost was high as there was a requirement of RFID reader. Also the architecture used was servlets technology which was not flexible as the clients of only java technology could be interfaced.

Taking all this drawbacks of existing system we have introduced the concept of NFC and cloud server. By using NFC tags, the cost of using RFID reader is cut down. And also by using cloud server i.e web server we can interface clients of any technology with the server.

\section{PROPOSED SYSTEM 3.1 Problem Statement}

"To develop an automated system using smart phone which will automatically monitor patients status and generate alerts and maintain logs on cloud server."

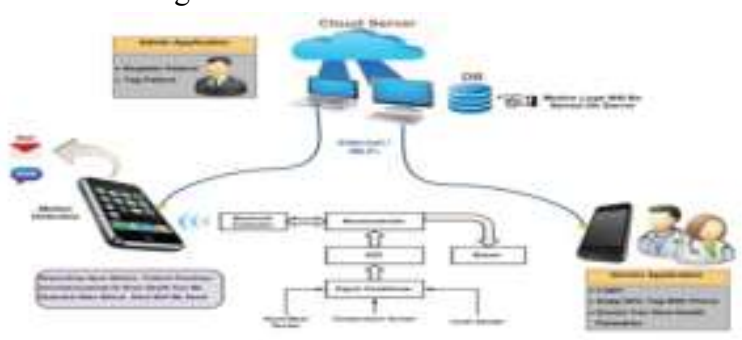

Figure 1: Proposed System Architecture

\subsection{Proposed Architecture}

The proposed architecture consist of various application Viz Admin application, doctor application, android application and cloud server. There is a hardware device connected to the android application through Bluetooth. The hardware device consist of sensors connected to it. In this paper we will be using basically 3 sensors i.e heart rate sensor, body temperature sensor and level sensor [saline level].

\section{Patient Position Sensor :}

The Patient Position Sensor (Accelerometer) monitors five different patient positions (prone, supine, standing/sitting, left and right.) In many cases, it is necessary to monitor the body positions and movements made because of their relationships to particular diseases (i.e., sleep apnea and restless legs syndrome). The body position sensor could help also to detect fainting or falling of elderly people or persons with disabilities and also detect death status through a motion detector.

\section{Body Temperature Sensor :}

The commonly accepted average core body temperature (taken internally) is $37.0^{\circ} \mathrm{C}\left(98.6^{\circ} \mathrm{F}\right)$. In healthy adults, body temperature fluctuates about $0.5^{\circ} \mathrm{C}\left(0.9^{\circ} \mathrm{F}\right)$ throughout the day, with temperatures being lower in the morning and higher in late afternoon and evening depending on the body's needs and activities the temperature change. Measuring body temperature is of great medical importance.The reason is that a by characteristic change in body temperature a number of diseases are accompanied. Likewise, by measuring body temperature, the course of certain diseases can be monitored, and the efficiency of a treatment initiated can be evaluated by the physician.

\section{Heart Rate Sensor :}

A normal resting heart rate for adult's ranges from 60 to 100 beats a minute. Generally, a lower heart rate at rest implies more efficient heart function and better cardiovascular fitness. The Heart Rate speeds up or slows down in a number of ways Bradycardia is defined as a resting heart rate below $60 \mathrm{bpm}$. 
However, heart rate from 50 to $60 \mathrm{bpm}$ are common among healthy people and do not necessarily require special attention. Tachycardia is defined as a resting heart rate above $100 \mathrm{bpm}$, though persistent rest rates between 80-100 bpm, mainly if they are present during sleep, may be signs of hyperthyroidism or anemia.

The number of sensors can be increased upto eight. The hardware device consist of a number of components i.e Bluetooth controller, microcontroller, ADC(Analog to Digital), signal conditioner and buzzer. The sensors are connected to the signal conditioner through which all the values of the sensors are collected and then sent to the ADC where the signal is converted from analog to digital and then sent to the microcontroller which transmits the data to the android application through a Bluetooth controller. In case of emergency the buzzer will ring or an SMS/Email will be sent to the doctor on the smart phone.

All the data and information related to the patient is stored on the cloud server. We have introduced cloud server so that clients of different platform can be interfaced which overcomes the disadvantage of using servlets.

Patient will be monitored for unconsciousness or death status through the phone camera. The state of the patient will be recorded and sent after a fixed time interval and in case of any emergency the buzzer will ring and also alerts will be sent through SMS or Email.

NFC tags will be used with the help of an NFC enabled smart phone by the doctor to retrieve the patient's information. By using NFC tags we are eliminating the paper work that had to be done during the registration of the patient when he/she is admitted

\section{PROPOSED ARCHITECTURE IMPLEMENTATION}

When a patient is admitted in hospital for the first time a unique id is provided to patient. NFC tag will be associated with every patient. equipped with NFC enabled smart phones will be equipped to Doctors and other staff. There will be an NFC issue app which will be used to read the content from mobile to NFC tag. Administrator can write unique tag id and application link in NFC tag by using the NFC issue app. To create patient application link administrator use IP of the server and then transfer to mobile by using same IP. Whenever NFC tag is placed near NFC enable smartphones the patient data is retrieved directly from the server. Doctor can also do the same thing to retrieve information of the patient from the server.

After the patient is admitted, android phone will be associated which will have motion detection to detect the death/ unconsciousness of the patient. Also there will be different sensors that will monitor different parameters such as heart beat rate, body temperature and level sensor (saline level). after a time interval the data will be transmitted through Bluetooth to the android phone which will further be sent to the cloud server through WiFi and stored there. The doctor can view the data any time and also can be viewed in graphical form.

\section{CONCLUSION}

In this proposed system, we have overcome the disadvantages of using RFID system by using NFC tags. We have also replaced the servlets architecture by using web service architecture. Hence the patients status is automatically generated and stored in the cloud server and also alerts are generated if the patient is in a critical condition. Currently it is possible to conclude that for providing a better solution to the m-healthcare hospital management system in many hospitals NFC promises appear to be credible.

\section{ACKNOWLEDGMENTS}

I am thankful to my guide Mrs. S.S.Shaikh for her complete support and guidance throughout the work.

\section{REFERENCES}

[1] J. Zhou, Xiaodong Lin, et.al. "PSMPA: Patient Selfcontrollable \& Multi-level Privacy Preserving cooperative authentication in distributed $m$-healthcare cloud computing system" in IEEE transaction on parallel and distributed systems, vol. 26, No. 6, June 2015, Pg. 1693-1703.

[2] Ran Wei, "Zhimin Yang", "Design and Implementation of Doctor-Patient system based on android" in 2012 international symposium on information technology in medicine and education(2012), Pg. 580-583.

[3] Ying-Wei Bai, Wen-Tai and You-Wei Chen, "Design and implementation of an embedded monitor system for detection of a patient's breath by double webcams" in medical measurements \& applications proceedings(MeMeA), 2010 IEEE international workshop on april 30-may 1 2010, Pg. 171-176.Tavel, P. 2007 Modeling and Simulation Design. AK Peters Ltd.

[4] J. Mirkovic, H. Bryhmi and C.M. Ruland, "A framework for the development of Ubiquitous patient support system", in pervasive health 2012, May 21-24, San Diego, United States, Pg. 81-88.

[5] Ms. Sohate R. Pawara, "Instant notification system in heterogeneous network with deployment of XMPP protocol" in Cloud \& Ubiquitous Computing \& Emerging Technologies(CUBE), 2013 international conference on 15-16 Nov, 2013, Pg. 87-92.

[6] P. Khanja, S. Wattanasirichaigan, Et.Al.,"A web base system for ECG data Transferred using Zigbee/IEEE technology" in the $3^{\text {rd }}$ international symposium on Biomedical engineering(ISBME 2008) Pg. 109-112.

[7] Mehmet R., Yuce Peng Choong Ng and Jamil Y. Khan "Monitoring of Physiological parameters from multiple patients using wireless sensor network" in Journal of Medical systems, vol. 32, IS. 5,PP 433-441

[8] AV.Gopi Krishna, C.Sreevardhan, S.Karun, S.P.Kumar,'NFC-based hospital Real-Time Patient management system" in IJETT- Vol.4 IS 4- April 2013 Pg. 626-629. 
International Journal of Computer Applications Technology and Research

Volume 4- Issue 12, 956 - 959, 2015, ISSN: 2319-8656

[9] S.Challa, G.Geethakumari, CSN Prasad,"Patient Data Viewer: An Android application for healthcare" in India Conference(INDICON), 2011 Annual IEEE, 16-18 Dec 2011.

[10] Lei Clifton, D.A.Clifton, M.A.F.Pimentel, Peter J.

WAtlinson and L. Tarassenko, "Predictive Monitoring of Mobile patients by combining clinical observations with Data from wearable sensors" in IEEE Journal of Biomedical and Health Informatics, Vol. 18, No 3, May 2014 Pg. 722-730. 